\title{
Pacific
}

Journal of

Mathematics

\section{PRIMITIVE IDEALS AND DERIVATIONS ON NONCOMMUTATIVE BANACH ALGEBRAS}

MARK PHILLIP THOMAS

Volume 159 No. 1 


\title{
PRIMITIVE IDEALS AND DERIVATIONS ON NON-COMMUTATIVE BANACH ALGEBRAS
}

\author{
MARC P. Thomas
}

\begin{abstract}
The Singer-Wermer Conjecture states that if $D$ is a (possibly unbounded) derivation on a commutative Banach algebra then the range of $D$ is contained in the (Jacobson) radical of the algebra. This conjecture is now known to be true. However, it is still not currently known whether or not the Singer-Wermer Conjecture on derivations extends to non-commutative Banach algebras in the following sense: if $D$ is a (possibly unbounded) derivation then is $D(P) \subseteq P$ for all primitive ideals $P$ of the algebra? This has become known as the non-commutative version of the Singer-Wermer Conjecture. We first correct an automatic continuity result in the literature concerning which (and how many) primitive ideals can fail to be invariant. Using this result together with some representation theory we prove a theorem about derivations whose second iteration annihilates some element (specifically, $D^{2} a=0$ implies that $D a$ is quasinilpotent). This theorem does not require commutativity of the algebra and it is easily seen to imply the Singer-Wermer Conjecture. The proof itself is done by contradiction in which the remaining case leads to a new derivation on a commutative subalgebra, and this case can be contradicted by the arguments used in the proof of the Singer-Wermer Conjecture.
\end{abstract}

1. Automatic continuity preliminaries. The fundamental work which started investigation into the ranges of derivations on Banach algebras is due to Singer and Wermer [8] in 1955. In this paper, the authors proved that every bounded derivation on a commutative Banach algebra mapped into the (Jacobson) radical. They also made a very insightful conjecture, namely that the assumption of boundedness was unnecessary. This became known as the Singer-Wermer Conjecture and was proved in 1987 by the author [9]. The arguments used in [9] are rather dependent on the commutativity of the Banach algebra.

In this paper we seek a proof of a result (Theorem 2.9) about derivations on (possibly non-commutative) Banach algebras which implies the Singer-Wermer Conjecture. In results of this type, a major obstacle is the discontinuity of the derivation. This was shown very early on in the reductions (which were needed for [9]) of the problem by Johnson [2] and Johnson and Sinclair [3] who also established a number of fundamental principles in what is now known as the theory 
of Automatic Continuity. In contrast, the case where the derivation is bounded is much better known. See the paper [5] by Mathieu and Murphy for a thorough and elegant discussion of related results (including the bounded version [5, Theorem 2.1] of our Theorem 2.9) in this case.

Hence, in our situation, it is not surprising that in order to prove our main result, Theorem 2.9 , we first require a number of results from Automatic Continuity. These results will be applied (in a noncommutative setting) in order to establish Proposition 1.10 which is fundamental to our argument in $\S 2$. Although this proposition appears in the literature in a paper of Jiang [1, Theorem 3.6], there is an error in both the statement and proof of the first step [1, Theorem 2.1] of his argument. We therefore believe that it is important to establish a correct proof of Proposition 1.10 and our approach in $\S 1$ will follow Jiang's strategy, which is certainly sound. It should also be noted that several important and supporting lemmas are essentially results of Johnson, Sinclair, and Laursen [2], [3], [7], [4] and we have noted their contributions below. In essence, Proposition 1.10 is very much a group accomplishment due to the work of Johnson, Sinclair, Laursen, and Jiang.

After Proposition 1.10 has been proved we will use representation theory and proof by contradiction to establish our main result (Theorem 2.9) in $\S 2$. In the remaining case of the proof we will see that it is possible to construct a new derivation on a commutative subalgebra, and it is then easy to finish off the proof. A real problem, at least for us, with attempting a proof of the so-called non-commutative Singer-Wermer conjecture (which states that there are actually no exceptional primitive ideals in the statement of Proposition 1.10 and which remains unproven) is that the assumption of a non-invariant primitive ideal seems too weak to enable one to do the type of reductions of the problem which we do in $\S 2$.

We will adhere to the following notation. We let $\mathscr{A}$ be a fixed Banach algebra over the complex field. It is not assumed to be commutative nor to have an identity element. We will let $\mathscr{R}$ denote the Jacobson radical of $\mathscr{A}$ so that $\mathscr{R}$ is the intersection of the kernels $P$ of all strictly irreducible representations $\pi$ of $\mathscr{A}$. Such ideals $P$ are denoted primitive ideals and it is well known that they can equivalently be defined in several other ways (see Rickart's book [6, Chapter 2] for the general representation theory of Banach algebras). Primitive ideals are necessarily closed. Results of Johnson [2] show that any 
associated strictly irreducible representation $\pi$ can be assumed to be continuous. For our purposes, a derivation $D$ will be a $\mathbb{C}$-linear map from $\mathscr{A}$ to itself satisfying

$$
D(x y)=x D y+(D x) y,
$$

for all $x, y \in \mathscr{A}$, and we will suppose that $D$ is fixed for the duration of this discussion. If $I$ is any closed (2-sided) ideal of $\mathscr{A}$ we will let $Q_{I}$ denote the canonical quotient map from $\mathscr{A}$ onto $\mathscr{A} / I$. The derivation $D$ itself is, of course, not assumed to be continuous, and we can measure its discontinuity by considering the so-called separating spaces of its powers:

$$
\mathscr{S}\left(D^{k}\right) \equiv\left\{x \in \mathscr{A} \mid \text { there exists } x_{n} \rightarrow 0 \text { and } D^{k}\left(x_{n}\right) \rightarrow x\right\},
$$

for each $k \in \mathbf{N}$. It is routine that each $\mathscr{S}\left(D^{k}\right)$ is a closed subspace of $\mathscr{A}$ and that if $R$ is any continuous linear operator with domain $\mathscr{A}$ then $R D^{k}$ is continuous if and only if $R\left(\mathscr{S}\left(D^{k}\right)\right) \equiv\{0\}$.

The following lemma is due to Johnson and Sinclair and Jiang [1, Lemma 3.5].

LEMMA 1.1. Let I be any closed ideal of $\mathscr{A}$ such that $Q_{I} D^{n}$ is continuous for all $n \in \mathbf{N}$. Then there exists a positive real constant $C$ such that

$$
\left\|Q_{I} D^{n}\right\| \leq C^{n}
$$

for all $n \in \mathbf{N}$.

Proof. Note that the hypothesis is equivalent to stating that $\mathscr{S}\left(D^{n}\right)$ $\subseteq I$ for all $n \in \mathbf{N}$. Define the linear subspace

$$
J \equiv\left\{x \in \mathscr{A} \mid Q_{I} x=0 \text { and } Q_{I} D^{n} x=0 \text { for all } n \in \mathbf{N}\right\} .
$$

Certainly $J \subseteq I$ and $J$ is closed as a consequence of the continuity of $Q_{I}$ and each $Q_{I} D^{n}, n \in \mathbf{N}$. Let $x \in J$ and $y \in \mathscr{A}$ and note that

$$
D^{k}(y x)=\left(D^{k} y\right) x+\sum_{l=0}^{k-1}\left(\begin{array}{l}
k \\
l
\end{array}\right)\left(D^{l} y\right) D^{k-l} x .
$$

If we apply $Q_{I}$ to the above, we see that $Q_{I} D^{n}(y x)=0$ for all $n \in \mathbf{N}$. This shows that $J$ is a left ideal. A similar argument shows that it is a right ideal, and we can then conclude that $J$ is a closed (2-sided) ideal of $\mathscr{A}$. Let $y \in \mathscr{S}(D)$. There is then $x_{k} \rightarrow 0$ with $D\left(x_{k}\right) \rightarrow y$. But, for every $n \in \mathbf{N}$,

$$
Q_{I} D^{n}(y)=\lim _{n \rightarrow \infty} Q_{I} D^{n+1}\left(x_{k}\right)=0,
$$


since each $Q_{I} D^{n+1}$ is continuous. Therefore, $\mathscr{S}(D) \subseteq J$, from which it follows that $Q_{J} D$ is continuous. Since it is clear that $D(J) \subseteq J$, $Q_{J} D$ factors through $\mathscr{A} / J$ as $\widehat{D} Q_{J}$ where $\widehat{D}$ is continuous and is, as usual, defined by $\widehat{D}(y+J)=D y+J$. This shows that we can also factor $Q_{I} D^{n}$ as $\Phi \widehat{D}^{n} Q_{J}$ where $\Phi$ is the canonical inclusion from $\mathscr{A} / J$ onto $\mathscr{A} / I$ (which exists since $J \subseteq I$ ). We therefore conclude that

$$
\left\|Q_{I} D^{n}\right\| \leq\|\widehat{D}\|^{n}
$$

for all $n \in \mathbf{N}$, since the other maps are norm depressing.

The next lemma is due to Johnson and Sinclair.

Lemma 1.2. Let $P$ be a primitive ideal of $\mathscr{A}$. If there exists a positive real constant $C$ such that

$$
\left\|Q_{P} D^{n}\right\| \leq C^{n}
$$

for all $n \in \mathbf{N}$, then $D(P) \subseteq P$.

Proof. Note that the quotient algebra $\mathscr{A} / P$ is a primitive algebra (it has one unique, up to isomorphism, faithful strictly irreducible representation) and is hence semi-simple. Let $y \in \mathscr{A}$ and $x \in P$ and observe that

$$
y D x=D(y x)-(D y) x \in D(P)+P .
$$

This shows that $(D(P)+P) / P$ is a left ideal of $\mathscr{A} / P$ (and a right ideal by analogous reasoning). Let $n \in \mathbf{N}$ and $x \in P$ and observe that

$$
D^{n}\left(x^{n}\right) \in n !(D x)^{n}+P,
$$

and hence

$$
(n !)^{1 / n}\left\|\left(\left(Q_{P} D x\right)^{n}\right)\right\|^{1 / n}=\left\|Q_{P} D^{n}\left(x^{n}\right)\right\|^{1 / n} \leq C\left\|x^{n}\right\|^{1 / n} .
$$

Since $C\left\|x^{n}\right\|^{1 / n}$ is bounded and since $(n !)^{1 / n} \rightarrow+\infty$, this shows that $Q_{P} D x$ is quasinilpotent. Since $x$ was an arbitrary element of $P$ it follows that $(D(P)+P) / P$ is a quasinilpotent left (and right) ideal of $\mathscr{A} / P$. Semi-simplicity forces $D(P) \subseteq P$.

The next important proposition is incorrectly stated in [1, Theorem 2.1] (the 9th line from the bottom of the page is unjustified; it is necessary to use the products $T_{1} T_{2} \cdots T_{m}$ rather than just the individual $T_{m}$ 's) but the basic principle was noted earlier and proved in a slightly different but equivalent formulation by Laursen [4, Proposition 2.1]. 
Proposition 1.3. Let $\left\{T_{i}\right\}_{i=1}^{\infty}$ be a sequence of continuous linear operators defined from $\mathscr{A}$ to itself and let $\left\{R_{i}\right\}_{i=1}^{\infty}$ be a sequence of continuous linear operators whose domain is $\mathscr{A}$ (but which may map onto other Banach spaces). Let $S$ be a (possibly discontinuous) linear map from $\mathscr{A}$ to itself. If

$$
R_{n} S T_{1} T_{2} \cdots T_{m} \text { is continuous for } m>n,
$$

then

$$
R_{n} S T_{1} T_{2} \cdots T_{n} \text { is continuous for sufficiently large } n \text {. }
$$

Proof. Without loss of generality, we may suppose that the norms of all the $T_{n}$ 's and $R_{n}$ 's are bounded by 1 . Let $U_{n}=T_{1} T_{2} \cdots T_{n}$ and suppose that the result fails. It is then possible to choose a strictly increasing sequence $\left\{n_{i}\right\} \subseteq \mathbf{N}$ and elements $\left\{a_{i}\right\}_{i=1}^{\infty} \subseteq \mathscr{A}$ such that $\left\|a_{i}\right\|<2^{-i}$ satisfies

$$
\left\|a_{i}\right\| \leq 2^{-i} \min \left\{\left\|R_{n_{j}} S U_{n_{l}}\right\|^{-1} \mid \text { for all } j<l \leq i\right\},
$$

and

$$
\left\|R_{n_{i}} S U_{n_{i}} a_{i}\right\| \geq 1+i+\left\|S \sum_{j=1}^{i-1} U_{n_{j}} a_{j}\right\|,
$$

for all $i \in \mathbf{N}$. Now let $a=\sum_{j=1}^{\infty} U_{n_{j}} a_{j} \in \mathscr{A}$ and note that for each $i \in \mathbf{N}$

$$
\begin{aligned}
\|S a\| \geq & \left\|R_{n_{i}} S a\right\| \\
\geq & \left\|R_{n_{i}} S U_{n_{i}} a_{i}\right\|-\left\|R_{n_{i}} S \sum_{j=1}^{i-1} U_{n_{j}} a_{j}\right\|-\left\|R_{n_{i}} S \sum_{j=i+1}^{\infty} U_{n_{j}} a_{j}\right\| \\
\geq & \left\|R_{n_{i}} S U_{n_{i}} a_{i}\right\|-\left\|S \sum_{j=1}^{i-1} U_{n_{j}} a_{j}\right\| \\
& -\left\|R_{n_{i}} S U_{n_{i+1}} \sum_{j=i+1}^{\infty} T_{n_{i+1}+1} \cdots T_{n_{j}} a_{j}\right\| \\
\geq & i+1-\left\|R_{n_{i}} S U_{n_{i+1}}\right\| \sum_{j=i+1}^{\infty}\left\|a_{j}\right\| \geq i,
\end{aligned}
$$

which, of course contradicts the fact that the norm is finite. This ends the proof of the proposition.

We now require a definition. 
Definition 1.4. Let $\mathscr{P}$ denote the set of all primitive ideals $P$ of $\mathscr{A}$ for which $Q_{P} D^{n}$ is continuous for all $n \in \mathbf{N}$.

From Lemma 1.1 and Lemma 1.2 it is clear that $P \in \mathscr{P}$ implies that $D(P) \subseteq P$. We will first show that $\mathscr{P}$ contains all primitive ideals of infinite codimension.

LEMMA 1.5 (Johnson and Sinclair). Let $\pi$ be a strictly irreducible representation of $\mathscr{A}$ on an infinite dimensional normed complex linear space $X$. Let $\left\{x_{i}\right\}_{i=0}^{\infty}$ be a linearly independent (over $\mathbb{C}$ ) subset of $X$. Then there exists a sequence $\left\{a_{i}\right\}_{i=1}^{\infty}$ in $\mathscr{A}$ such that

$$
\pi\left(a_{1}\right) x_{0}=0, \text { and }\left\{\pi\left(a_{1}\right) x_{1}, \pi\left(a_{1}\right) x_{2}, \ldots\right\}
$$

is linearly independent,

$$
\pi\left(a_{2} a_{1}\right) x_{0}=0, \pi\left(a_{2} a_{1}\right) x_{1}=0, \quad \text { and }\left\{\pi\left(a_{2} a_{1}\right) x_{2}, \pi\left(a_{2} a_{1}\right) x_{3}, \ldots\right\}
$$

is linearly independent, and so on; in general,

$$
\begin{aligned}
& \pi\left(a_{n} \cdots a_{2} a_{1}\right) x_{m}=0, \text { for } m<n, \quad \text { and } \\
& \left\{\pi\left(a_{n} \cdots a_{2} a_{1}\right) x_{n}, \pi\left(a_{n} \cdots a_{2} a_{1}\right) x_{n+1}, \ldots\right\}
\end{aligned}
$$

is linearly independent for all $n \in \mathbf{N}$.

Proof. Note that since $\mathscr{A}$ is a complex Banach algebra, the centralizer of $\mathscr{A}$ on $X$ is $\mathbb{C}$. Then apply the construction in [3, Theorem 2.2] (which is itself dependent upon induction on [3, Lemma 2.1]). This yields the desired sequence $\left\{a_{i}\right\}_{i=1}^{\infty}$.

The next result is essentially [1, Lemma 3.1] with a corrected proof (it is necessary to use the products $T_{1} T_{2} \cdots T_{m}$ rather than just the individual $T_{m}$ 's).

LEMMA 1.6. Let $\pi$ be an irreducible representation of $\mathscr{A}$ on an infinite dimensional normed complex linear space $X$. Then, for all $n \in \mathbf{N}$ and $x \in X$ the map

$$
b \rightarrow \pi\left(D^{n}(b)\right) x
$$

is continuous on $\mathscr{A}$.

Proof. Denote the above map by $\varphi_{n, x}$. If $\varphi_{n, x}$ is continuous for some $x \neq 0, x \in X$, then, given $y \in X$ choose (using the strict 
irreducibility of $\pi$ ) an element $c \in \mathscr{A}$ satisfying $\pi(c) x=y$. Then note that

$$
\begin{aligned}
\pi\left(D^{n}(b)\right) y & =\pi\left(D^{n}(b) c\right) x \\
& =\pi\left(D^{n}(b c)\right) x-\sum_{l=0}^{n-1}\left(\begin{array}{l}
n \\
l
\end{array}\right) \pi\left(D^{l}(b)\right) \pi\left(D^{n-l}(c)\right) x .
\end{aligned}
$$

This means that if the result fails it is possible to find a minimal index $k \in \mathbf{N}$ such that $\varphi_{l, x}$ is continuous for all $x \in X$ with $l<k$, and $\varphi_{k, x}$ is discontinuous for every non-zero $x \in X$. Since $X$ is infinite dimensional we can find a linearly independent subset $\left\{x_{i}\right\}_{i=0}^{\infty}$. Now apply Lemma 1.5 in order to obtain the sequence $\left\{a_{i}\right\}_{i=1}^{\infty} \subseteq \mathscr{A}$. Define, for $i=1,2, \ldots$, the continuous linear operators $T_{i}(b)=$ $b a_{i}, b \in \mathscr{A}$, and define, for $i=0,1, \ldots$, the continuous linear operators $R_{i}(b)=\pi\left(D^{k-1}(b)\right) x_{i}, b \in \mathscr{A}$. Then note that

$$
\begin{aligned}
R_{n} D & T_{1} T_{2} \cdots T_{m}(b)=\pi\left(D^{k-1}\left(D\left(b a_{m} \cdots a_{2} a_{1}\right)\right)\right) x_{n} \\
& =\pi\left(D^{k}\left(b a_{m} \cdots a_{2} a_{1}\right)\right) x_{n} \\
& =\pi\left(D^{k}(b)\right) \pi\left(a_{m} \cdots a_{2} a_{1}\right) x_{n}+\sum_{l=0}^{k-1}\left(\begin{array}{l}
k \\
l
\end{array}\right) \varphi_{l, v(l, n, m)}(b),
\end{aligned}
$$

where $v(l, n, m)=\pi\left(D^{k-l}\left(a_{m} \cdots a_{2} a_{1}\right)\right) x_{n}$, a vector in $X$ which does not depend on $b$. Since $\pi\left(a_{m} \cdots a_{2} a_{1}\right) x_{n}$ is the zero vector if $m>n$ but is non-zero if $m=n$, it follows that $R_{n} D T_{1} T_{2} \cdots T_{m}$ is a continuous function if $m>n$, but a discontinuous function if $m=n$. This contradicts the automatic continuity result of Lemma 1.3 , and the result follows.

COROllary 1.7. If $P$ is a primitive ideal in $\mathscr{A}$ of infinite codimension then $P \in \mathscr{P}$ and $D(P) \subseteq P$.

Proof. Let $P$ be a primitive ideal of infinite codimension in $\mathscr{A}$ and let $\pi$ be a corresponding strictly irreducible representation with kernel $P$ on a normed linear space $X$. It is clear that $X$ must be infinite dimensional; otherwise $\pi(\mathscr{A})$ would be isomorphic to a finite dimensional matrix ring. Lemma 1.6 implies that the map

$$
b \rightarrow \pi\left(D^{n}(b)\right) x
$$

is continuous for every $n \in \mathbf{N}$ and $x \in X$. Hence, we see that for $n \in \mathbf{N}$

$$
\mathscr{S}\left(D^{n}\right) \subseteq \bigcap_{x \in X}\{b \in \mathscr{A} \mid \pi(b) x=0\}=P .
$$


Therefore, it follows that $\mathscr{S}\left(D^{n}\right) \subseteq P$ for all $n \in \mathbf{N}$ and the result follows.

We next work on primitive ideals $P$ with finite codimension. Of course, this means that if $\pi$ is a corresponding strictly irreducible representation on a (complex finite dimensional) vector space $X$ with kernel $P$ then $\mathscr{A} / P \cong L(X)$, the full matrix ring of operators over $\mathbb{C}$. Hence the codimension of $P$ is the dimension of $L(X)$ which is the square of the dimension of the vector space $X$.

LEMMA 1.8 (Johnson and Sinclair). Let $\left\{\pi_{i}\right\}_{i=1}^{\infty}$ be non-equivalent strictly irreducible representations of $\mathscr{A}$ on finite dimensional vector spaces $X_{i}, i \in \mathbf{N}$. Then there exists a sequence $\left\{a_{i}\right\}_{i=1}^{\infty} \subseteq \mathscr{A}$ such that

$$
\pi_{n}\left(a_{m}\right)=0
$$

whenever $m>n, b u t$

$$
\pi_{n}\left(a_{m}\right) \text { is regular in } L\left(X_{n}\right) \text {, }
$$

if $m \leq n$.

Proof. This is precisely the statement of [3, Lemma 3.2].

The next result is essentially [1, Theorem 3.4], again with a corrected proof (it is necessary here as well to use the products $T_{1} T_{2} \cdots T_{m}$ rather than just the individual $T_{m}$ 's).

LEMMA 1.9. $\mathscr{P}$ contains all but possibly finitely many primitive ideals of finite codimension.

Proof. We suppose that the result fails. As a consequence of Corollary 1.7 , it is then possible to find a sequence of distinct primitive ideals $\left\{P_{i}\right\}_{i=1}^{\infty}$ of finite codimension in $\mathscr{A}$ which are not in $\mathscr{P}$. Furthermore, it must be the case that for each $P_{i}$ there is $k_{i} \in \mathbf{N}$ such that $Q_{P_{i}} D^{k}$ is discontinuous if $k=k_{i}$ but continuous if $k<k_{i}$. If, for each $i \in \mathbf{N}, \pi_{i}$ is an associated strictly irreducible representation of $\mathscr{A}$ on a vector space $X_{i}$ with kernel $P_{i}$ then it is clear that the $\left\{\pi_{i}\right\}_{i=1}^{\infty}$ are non-equivalent. Apply the previous lemma to obtain the sequence $\left\{a_{i}\right\}_{i=1}^{\infty}$. Also note that $\mathscr{A} / P_{i} \cong L\left(X_{i}\right)$. Define, for $i=1,2, \ldots$, the continuous linear operators $T_{i}(b)=b a_{i}, b \in \mathscr{A}$, and define, for $i=0,1, \ldots$, the continuous linear operators $R_{i}(b)=Q_{P_{t}} D^{k_{t}-1}(b)$, 
$b \in \mathscr{A}$. Then note that

$$
\begin{aligned}
R_{n} D T_{1} & T_{2} \cdots T_{m}(b)=Q_{P_{n}} D^{k_{n}}\left(b a_{m} \cdots a_{2} a_{1}\right) \\
= & \left(Q_{P_{n}} D^{k_{n}}(b)\right)\left(Q_{P_{n}}\left(a_{m} \cdots a_{2} a_{1}\right)\right) \\
& +\sum_{l=0}^{k_{n}-1}\left(\begin{array}{c}
k_{n} \\
l
\end{array}\right)\left(Q_{P_{n}} D^{l}(b)\right)\left(Q_{P_{n}} D^{k_{n}-l}\left(a_{m} \cdots a_{2} a_{1}\right)\right) .
\end{aligned}
$$

Since $Q_{P_{n}}\left(a_{m} \cdots a_{2} a_{1}\right)=0$ for $m>n\left(\right.$ as $\left.\pi_{n}\left(a_{m}\right)=0\right)$ and since

$$
Q_{P_{n}}\left(a_{n} \cdots a_{2} a_{1}\right)=Q_{P_{n}}\left(a_{n}\right) \cdots Q_{P_{n}}\left(a_{2}\right) Q_{P_{n}}\left(a_{1}\right),
$$

which is regular (as $\pi_{n}\left(a_{i}\right)$ is regular for $\left.i=1,2, \ldots, n\right)$, it follows that $R_{n} D T_{1} T_{2} \cdots T_{m}$ is continuous for $m>n$ and discontinuous for $m=n$. Again, this contradicts Lemma 1.3. Therefore $\mathscr{P}$ must be a finite set and its only elements can be primitive ideals with finite codimension.

The next result is immediate.

Proposition 1.10. It must be the case that $Q_{P} D^{n}$ is continuous for all $n \in \mathbf{N}$ and $D(P) \subseteq P$ for all primitive ideals $P$ of $\mathscr{A}$ except possibly finitely many exceptional primitive ideals $P_{1}, P_{2}, \ldots, P_{n}$ which must necessarily be of finite codimension.

2. Proof of the Main Theorem. Now that we have established Proposition 1.10 , we continue to assume that $\mathscr{A}$ is a (possibly non-commutative) Banach algebra, $D$ is an (unbounded) derivation from $\mathscr{A}$ to itself, and we let $a$ be any element of $\mathscr{A}$ satisfying $D^{2} a=0$. We first note that the following proposition is an immediate consequence of the well-known result [6, Theorem 2.2.9, page 54] that $\pi(r)$ quasiregular for all strictly irreducible representations $\pi$ of $\mathscr{A}$ implies that $r$ itself is quasi-regular.

Proposition 2.1. If $\mathscr{L}$ is a set representing all non-isomorphic strictly irreducible representations $\pi$ of $\mathscr{A}$ then for any element $b \in$ $\mathscr{A}$,

$$
\sigma(b) \subseteq\left(\bigcup_{\pi \in \mathscr{L}} \sigma(\pi(b))\right) \cup\{0\}
$$

Let $P$ be one of the non-exceptional primitive ideals which satisfy the conclusions of Proposition 1.10 and let $\pi$ be an associated 
strictly irreducible representation with kernel $P$. Then $D$ drops to a continuous derivation $\dot{D}$ on the quotient $\mathscr{A} / P$ via

$$
\dot{D} \dot{x} \equiv(\dot{D} x),
$$

where the $d o t$ denotes the coset in $\mathscr{A} / P$. Note that $\dot{D}^{k}(\dot{a})=\dot{0}$ for all $k \in \mathbf{N}, k \geq 2$ and therefore

$$
\begin{aligned}
\left\|(\dot{D} \dot{a})^{n}\right\|^{1 / n} & =\left\|(1 / n !) \dot{D}^{n}\left(\dot{a}^{n}\right)\right\|^{1 / n} \\
& =(n !)^{-1 / n}\left\|\dot{D}^{n}\left(\dot{a}^{n}\right)\right\|^{1 / n} \\
& \leq(n !)^{-1 / n}\|\dot{D}\|\left\|\dot{a}^{n}\right\|^{1 / n}
\end{aligned}
$$

and this latter term tends to 0 as $n \rightarrow \infty$. Hence, $D a+P$ is quasinilpotent in $\mathscr{A} / P$ and therefore the spectrum $\sigma(\pi(D a))=\{0\}$. Since only finitely many primitive ideals may be exceptional in not satisfying the conclusions of Proposition 1.10, and since, for these ideals, the codimension is finite, Proposition 2.1 immediately implies the following corollary.

COROLlaRY 2.2. If $D^{2} a=0$ then the spectrum $\sigma(D a)$ is a finite set.

For the next lemma, we first require a definition.

Definition 2.3. Let $S$ be any subset of $\mathscr{A}$. Define the commutant of $S$ as follows.

$$
C(S) \equiv\{x \in \mathscr{A} \mid x s=s x \text { for all } s \in S\} .
$$

LemMA 2.4. Let $S$ be any subset of $\mathscr{A}$ satisfying $D(S) \subseteq S$. Then $D(C(S)) \subseteq C(S)$.

Proof. Let $x \in C(S)$, so that $x s=s x$ for all $s \in S$. Then $D(x s)=$ $D(s x)$ for all $s \in S$, from which it follows that

$$
x D s+(D x) s=s D x+(D s) x,
$$

for all $s \in S$. Since $D s \in S$ for all $s \in S$, the above equation reduces to $(D x) s=s D x$, for all $s \in S$. Therefore we see that $D x \in C\left(S^{3}\right)$ also.

Lemma 2.5. Let $S$ be any subset of $\mathscr{A}$. Then $C(S)$ is a closed subalgebra of $\mathscr{A}$. If furthermore $S$ itself is a commutative subset of 
$\mathscr{A}$ then $C(S) \cap C(C(S))$ is a closed commutative subalgebra of $\mathscr{A}$ containing $S$.

Proof. It is routine to check the above assertions.

Returning to $\sigma(D a)$, suppose that there exists $\lambda \neq 0, \lambda \in \sigma(D a)$. We fix such a $\lambda$ for the following discussion and we seek a contradiction. Since $\sigma(D a)$ is a finite set there is a function $f$ analytic on a neighborhood of $\sigma(D a)$ which is identically 1 on a neighborhood of $\{\lambda\}$ but identically 0 on a neighborhood of $\sigma(D a) \sim\{\lambda\}$ (e.g. we could explicitly take $f$ to be the uniform limit of the analytic functions $f_{m}(\zeta)$ where $f_{m}(\zeta)=\left(1-K^{m}(\zeta-\lambda)^{m}\right)^{-1}$ for a sufficiently small choice of $K$ on a suitable disconnected neighborhood of $\sigma(D a))$. Hence, $e=f(D a)$ is an idempotent which commutes with $D a$. Since $e$ is an analytic function of $D a, e$ also commutes with any element of $\mathscr{A}$ which commutes with $D a$. Furthermore, $e \mathscr{A} e$ is a closed subalgebra of $\mathscr{A}$ with identity element $e$ and with an element $e(D a) e=e D a$ which satisfies

$$
\sigma_{e \mathscr{A} e}(e(D a) e)=\sigma_{e \mathscr{A} e}(e D a)=\{\lambda\} .
$$

Since $e$ is the identity element of $e \mathscr{A} e$ it is also clear that

$$
\sigma_{e \mathscr{A} e}(e(D a) e-\lambda e)=\{0\} .
$$

We are now ready to prove the following lemma.

LEMMA 2.6. Let $\lambda \neq 0$ be an element of $\sigma(D a)$. Then there is an idempotent $e \in \mathscr{A}$ which commutes with $\mathrm{Da}$ and which commutes with anything that commutes with $\mathrm{Da}$ and satisfies the following:

(i) The element $s=(e(D a) e-\lambda e)$ is quasinilpotent and satisfies $D s=0$.

(ii) $D(e a e)=\lambda e+a$ and $D^{2}(e a e)=0$.

Proof. Let $e=f(D a)$ as above. We have noted that

$$
\sigma_{e \mathscr{A} e}(e(D a) e-\lambda e)=\{0\} .
$$

There is thus a quasinilpotent element $s$ of $e \mathscr{A} e$ such that $e(D a) e=$ $\lambda e+s$ (certainly $s$ is quasinilpotent in $\mathscr{A}$ also, although $\mathscr{A}$ need not have an identity element). Let $S$ be the (non-closed) commutative subalgebra generated by $D a$. Since $D^{2} a=0$ it is clear that $D(S) \equiv$ $\{0\}$ so that $D(S) \subseteq S$. Next note that since $D e=e D e+(D e) e$ and since $e \in C(S) \cap C(C(S))$, Lemma 2.4 implies that $D e$ is in the closed 
commutative subalgebra $C(S) \cap C(C(S))$ also. Since $e$ is idempotent and since $e$ and $D e$ commute, it follows that

$$
e(D e)=e(e D e+(D e) e)=e(2 e D e)=2 e(D e),
$$

so that $e D e=0$. Therefore we see that

$$
D e=e D e+(D e) e=2 e D e=0,
$$

as well. It is then routine to compute the following:

$$
\begin{aligned}
D(e a e) & =(D e) a e+e(D a) e+e a D e \\
& =0+e(D a) e+0=\lambda e+s,
\end{aligned}
$$

and

$$
\begin{aligned}
D^{2}(e a e) & =D(e(D a) e) \\
& =D e(D a) e+e\left(D^{2} a\right) e+e D a D e=0 .
\end{aligned}
$$

Since $D e=0$, it is also the case that $D s=0$, and this completes the proof of the lemma.

We now focus our attention on the closed subalgebra $e \mathscr{A} e$ and we define a new derivation $D_{1}$ on this unital Banach algebra as follows:

$$
D_{1}(\text { exe }) \equiv e D(\text { exe }) e,
$$

for $x \in \mathscr{A}$. It is routine to check that $D_{1}$ is a derivation. Since $D e=0$ it is also routine to establish that

$$
D_{1}(\text { exe })=e(D x) e .
$$

We have the following lemma concerning $D_{1}$.

LeMma 2.7. Let $\lambda, e$, and $s$ be as in Lemma 2.6, and define $D_{1}$ on eAde via $D_{1}($ exe $)=e(D x) e$, for $x \in \mathscr{A}$. Then there exists an element $t$ in e⿻lA $e$ satisfying

$$
D_{1} t=e \quad \text { and } \quad D_{1}^{2} t=0 .
$$

Proof. First note that

$$
D_{1}(e a e)=e D(e a e) e=e(\lambda e+s) e=\lambda e+s .
$$

Since $D_{1}(e)=e D(e) e=0$ and $D_{1}(s)=e D(s) e=0$ it is clear that $D_{1}^{2}(e a e)=0$. Since $\lambda \neq 0$, since $e$ is an identity element for $e \mathscr{A} \dot{e}$, and since $s$ is quasinilpotent, it follows that $(\lambda e+s)^{-1}$ exists in $e \mathscr{A} e$. Applying $D_{1}$ to the equation $(\lambda e+s)(\lambda e+s)^{-1}=e$, we see that

$$
\left(D_{1}(\lambda e+s)\right)(\lambda e+s)^{-1}+(\lambda e+s) D_{1}\left((\lambda e+s)^{-1}\right)=0,
$$


from which it follows that

$$
(\lambda e+s) D_{1}\left((\lambda e+s)^{-1}\right)=0,
$$

and, since $(\lambda e+s)$ is invertible,

$$
D_{1}\left((\lambda e+s)^{-1}\right)=0 .
$$

Now define $t=(e a e)(\lambda e+s)^{-1}$, which is certainly an element of $e \mathscr{A} e$, and note that

$$
D_{1}(t)=e,
$$

since $D_{1}\left((\lambda e+s)^{-1}\right)=0$. This forces

$$
D_{1}^{2}(t)=D_{1}(e)=0,
$$

and ends the proof of the lemma.

LEMMA 2.8. Let $e$ and $t \in e \mathscr{A} e$ be as in Lemma 2.7. Define $T$ to be the commutative (non-closed) unital subalgebra generated by $e$ and $t$ in eAde. Also define

$$
\mathscr{A}_{t} \equiv C(T) \cap C(C(T)),
$$

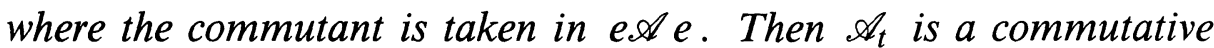
unital Banach algebra containing $e$ and $t$ which is invariant under the derivation $D_{1}$ and this derivation maps $t$ to the identity element $e$.

Proof. Since $T$ is a commutative subalgebra it is clear upon applying Lemma 2.5 that $\mathscr{A}_{t}$ is a closed commutative subalgebra of $e \mathscr{A} e$. It is unital and contains $t$ since $T \subseteq \mathscr{A}_{t}$. Since $T$ is clearly invariant under $D_{1}$, it is clear that $\mathscr{A}_{t}$ is invariant under $D_{1}$ as a consequence of Lemma 2.4. Finally, $D_{1}(t)=e$ by our above computation.

We have now contradicted the traditional Singer-Wermer Conjecture [9] since we have a commutative Banach algebra $\mathscr{A}_{t}$ with a derivation $D_{1}$ and an element $t$ such that $D_{1}(t) \notin$ radial $\left(\mathscr{A}_{t}\right)$ as the identity element $e$ is surely not in the radial of $\mathscr{A}_{t}$. This means that our original hypothesis of $\lambda \neq 0, \lambda \in \sigma(D a)$ must be false. We have therefore proved

THEOREM 2.9. Let $\mathscr{A}$ be a (possibly non-commutative) Banach algebra and let $D$ be a (possibly unbounded) derivation from $\mathscr{A}$ to itself. If $a \in \mathscr{A}$ and

$$
D^{2} a=0 .
$$

Then $\sigma(D a)=\{0\}$. 
We note in passing that it is easy to show that the truthfulness of Theorem 2.9 implies the truthfulness of the Singer-Wermer Conjecture since the assumption of commutativity and a derivation which doesn't map into the radical together with Johnson's reduction of the Conjecture [2] produces a derivation on a commutative radical Banach algebra with identity adjoined which does not leave the radical itself invariant. Since we are in a commutative situation, it is then easy to modify this derivation (multiplying by a suitable invertible element) and produce a new derivation which maps an element $r$ of the radical to the identity element. Again, using commutativity, $D^{2} r=0$ since $\mathrm{Dr}$ is the identity element, and this, of course, would violate Theorem 2.9 .

\section{REFERENCES}

[1] X. Jiang, Remarks on automatic continuity of derivations and module derivations, Acta Math. Sinica, New Series, 4 (3) (1988), 227-233.

[2] B. E. Johnson, Continuity of derivations on commutative algebras, Amer. J. Math., 91 (1969), 1-10.

[3] B. E. Johnson and A. M. Sinclair, Continuity of derivations and a problem of Kaplansky, Amer. J. Math., 90 (1968), 1067-1073.

[4] K. B. Laursen, Some remarks on automatic continuity, Lecture Notes in Math., vol. 512, Springer-Verlag, Berlin and New York, 1976, pp. 96-108.

[5] M. Mathieu and G. J. Murphy, Derivations mapping into the radical, Arch. Math., to appear.

[6] C. Rickart, General Theory of Banach Algebras, Krieger, 1974.

[7] A. M. Sinclair, Automatic continuity of linear operators, London Math. Soc. Lecture Note Series, vol. 21, Cambridge Univ. Press, Cambridge, 1976.

[8] I. M. Singer and J. Wermer, Derivations on commutative normed algebras, Math. Ann., 129 (1955), 260-264.

[9] M. P. Thomas, The image of a derivation is contained in the radical, Ann. of Math. (2), 128 (1988), 435-460.

Received September 9, 1991. The author was supported by NSF grant DMG 9002375.

California State University

BAKERSFIELD, CA 93311-1099 


\section{PACIFIC JOURNAL OF MATHEMATICS}

Founded by

E. F. BECKENBACH (1906-1982) F. Wolf (1904-1989)

\section{EDITORS}

\author{
V. S. VARADARAJAN \\ (Managing Editor) \\ University of California \\ Los Angeles, CA 90024-1555 \\ vsv@math.ucla.edu \\ F. Michael Christ \\ University of California \\ Los Angeles, CA 90024-1555 \\ christ@math.ucla.edu
}

\section{Herbert Clemens}

University of Utah

Salt Lake City, UT 84112

clemens@math.utah.edu

\author{
ThOMAs ENRIGHT \\ University of California, San Diego \\ La Jolla, CA 92093 \\ tenright@ucsd.edu \\ Nicholas Ercolani \\ University of Arizona \\ Tucson, AZ 85721 \\ ercolani@math.arizona.edu \\ R. FINN \\ Stanford University \\ Stanford, CA 94305 \\ finn@gauss.stanford.edu \\ VAUGHAN F. R. Jones \\ University of California \\ Berkeley, CA 94720 \\ vfr@math.berkeley.edu
}

STEVEN KeRCKHOFF

Stanford University

Stanford, CA 94305

spk@gauss.stanford.edu

Martin ScharlemanN

University of California

Santa Barbara, CA 93106

mgscharl@henri.ucsb.edu

Harold Stark

University of California, San Diego

La Jolla, CA 92093

\section{SUPPORTING INSTITUTIONS}

UNIVERSITY OF ARIZONA

UNIVERSITY OF BRITISH COLUMBIA

CALIFORNIA INSTITUTE OF TECHNOLOGY

UNIVERSITY OF CALIFORNIA

UNIVERSITY OF MONTANA

UNIVERSITY OF NEVADA, RENO

NEW MEXICO STATE UNIVERSITY

OREGON STATE UNIVERSITY
UNIVERSITY OF OREGON

UNIVERSITY OF SOUTHERN CALIFORNIA

STANFORD UNIVERSITY

UNIVERSITY OF HAWAII

UNIVERSITY OF UTAH

WASHINGTON STATE UNIVERSITY

UNIVERSITY OF WASHINGTON 


\section{PACIFIC JOURNAL OF MATHEMATICS}

Volume 159 No. $1 \quad$ May 1993

An application of the very weak Bernoulli condition for amenable groups

SCOT ROBERT ADAMS and JEFFREY EDWARD STEIF

An application of homogenization theory to harmonic analysis on solvable Lie groups of polynomial growth

G. Alexopoulos

The standard double soap bubble in $\mathbf{R}^{2}$ uniquely minimizes perimeter

Joel Foisy, Manuel Alfaro Garcia, JefFrey FARlowe

Brock, NiCKELOUS Hodges and JASON ZimbA

Pseudo regular elements and the auxiliary multiplication they induce

BARRY E. JOHNSON

A converse to a theorem of Komlós for convex subsets of $L_{1}$

CHRISTOPHER JOHN LENNARD

General Kac-Moody algebras and the Kazhdan-Lusztig conjecture

WAYNE L. NEIDHARDT

The flow space of a directed $G$-graph

WILLIAM LINDALL PASCHKE

Primitive ideals and derivations on noncommutative Banach algebras

MARK PHILLIP THOMAS

Equivariant Nielsen numbers

PETER N-S WONG

Volumes of tubular neighbourhoods of real algebraic varieties

RichaRd ALEXANDER WONGKEW

The intrinsic group of Majid's bicrossproduct Kac algebra 